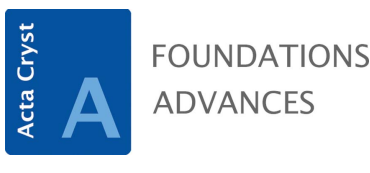

ISSN 2053-2733

\section{Quantum Theory of Materials. By Efthimios Kaxiras and John D. Joannopoulos. Cambridge University Press, 2019. Hardback, pp. 645. Price GBP 64.99, USD 89.99. ISBN 9780521117111.}

\author{
Mike Glazer*
}

Department of Physics, Oxford University, Parks Road, Oxford, OX1 3PU, UK. *Correspondence e-mail: mike.glazer@physics.ox.ac.uk

Keywords: book review; quantum theory; materials.

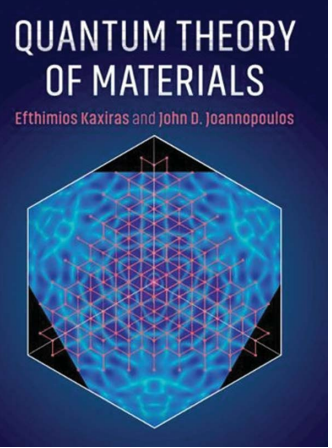

(C) 2020 International Union of Crystallography
This is a mighty tome with 645 pages of text, closely written and packed full of important concepts of prime concern to the theoretical physicist working in the field of condensed matter. It is beautifully produced with stunning diagrams, and covers all the material you would expect in such a text. The authors are Efthimios Kaxiras, who is John Hasbrouck Van Vleck Professor of Pure and Applied Physics at Harvard University, and John D. Joannopoulos, who is the Francis Wright Davis Professor of Physics at MIT. As you would expect coming from these two venerable institutions, the scientific level is very high indeed. This is not a book for the faint-hearted, as it requires much careful study to get through all of the material. Who is it aimed at? I would say graduate students in theoretical physics as well as those at a more senior level, but be prepared for a lot of study. It definitely reaches way above the heads of undergraduates.

Over a span of ten chapters, the authors start with bonding of atoms, followed by an introduction to translational periodicity, including Brillouin zones. This chapter contains a useful periodic table of elements with several physical properties and structure types. However, I noticed that there is no entry for polonium, the only crystalline material which at ambient conditions forms the unlikely 'simple' cubic structure that is so often taught in condensed matter texts. I much appreciated a figure which on the left showed a plot of the energy versus atomic separation for a metal, while on the right-hand side the corresponding energy-level diagram. This nicely illustrates how the energy levels which are degenerate at large distances start to split on reducing the atomic bond distances to form a band.

Anyway, on to a chapter curiously titled 'Symmetries beyond translational periodicity'. Reading this I realized that they meant the introduction of rotations, reflections, inversions etc. So this took them into concepts such as Bravais lattices, point groups and on to electronic band structures. Sadly, seen from a crystallographer's point of view, they use the Schoenflies notation, which, while it is OK for point groups, is quite useless in describing space groups. The authors say 'There is a somewhat more rational set of conventions for naming crystallographic point groups described in the International Tables for Crystallography. This scheme is more complicated.' (My emphasis.) Really? I think they really mean that neither of them is prepared to learn about the International Tables for Crystallography notation.

Anyway, proceeding further, we have chapters devoted to many-particle systems described in single-particle terms, and then electronic properties of crystals. I was especially interested here in the way they dealt with the electronic properties of benzene, where the molecule was treated effectively as being one-dimensional by thinking of the atoms lying on a circle and using periodic boundary conditions to create a set of effective wavevectors, in the same way we usually derive densities of states for electrons and phonons in a crystal. The result is an energy wavevector graph with solutions at $k=0$, $\pm 1 / 3, \pm 2 / 3$ and \pm 1 . This technique is then applied also to polyacetylene. This is very clever and illuminating.

This is followed by a chapter on electronic excitations and lattice vibrations and deformations (of course, we crystallographers know that lattices cannot vibrate or be deformed, don't we? But let's not go there). After this there is a section on phonon 
interactions and topological constraints, a very fashionable subject right now in theoretical and experimental condensed matter physics. Finally, there is a chapter on magnetic behaviour, going through the usual sequence of Pauli paramagnetism, Ising models and Heisenberg ferromagnets.

Throughout the book the chapters end with problems for the reader to solve, with solutions available online. A nice touch.

So, having said good things about this book, I am sorry to have to point to the usual mistakes where the crystallography is concerned. In my experience it is an uphill struggle to get theoretical physicists to take seriously crystallographic concepts and terminology, despite the fact that it is the crystallography that underpins most of solid-state physics. This spoils what in all other aspects is a fine textbook. As always with theoretical physics, and annoyingly to me, we have the usual confusions between lattices and crystal structure, spread throughout the book. They occur too numerously to list here, but consider just a few. We have the 'diamond lattice' when they mean the diamond structure. Statements like 'electrons are shared between all the atoms in a lattice' or 'charged ions which are repeated periodically to form a lattice'. We know that a lattice consists of points, not atoms, don't we? We get crystallographic horrors such as f.c.c. sublattices, interpenetrating cubic lattices and so on. Then there is the usual theorists' 'honeycomb lattice'. This is actually based on hexagonal symmetry in which the unit cell contains two atoms, unrelated by a lattice vector: in other words it is a structure, not a lattice. Again, there is mention of the h.c.p. lattice, which is simply a hexagonal lattice. H.c.p. refers to the packing of atoms, not lattice points. I could go on, but you get the idea.

In short then, this is a very good book if you have the stamina to wade through all of the solid-state theory in close detail, and important for anyone working in condensed matter theory. But do try to ignore, or better, correct for yourself, the crystallographic mistakes at the same time. 